\title{
Nonequilibrium dynamics of a system with quantum frustration
}

\author{
Heiner Kohler, ${ }^{1, *}$ Andreas Hackl, ${ }^{2}$ and Stefan Kehrein ${ }^{3}$ \\ ${ }^{1}$ Instituto de Ciencias Materiales de Madrid, CSIC, C/ Sor Juana Inés de la Cruz 3, 28049 Madrid, Spain \\ ${ }^{2}$ SAP AG, SAP Allee 45, 68789 St. Leon-Rot, Germany \\ ${ }^{3}$ Departement of Physics, Georg-August-Universität Göttingen, Friedrich-Hund Platz 1, 37077 Göttingen, Germany
}

(Received 6 April 2013; revised manuscript received 9 October 2013; published 15 November 2013)

\begin{abstract}
Using flow equations and equilibrium and nonequilibrium dynamics of a two-level system (TLS) is investigated, which couples Ohmically via noncommuting components to two independent oscillator baths. In equilibrium, the two-level energy splitting is protected when the TLS is coupled symmetrically to both baths. A critical asymmetry angle separates the localized from the delocalized phase. Real-time decoherence of a nonequilibrium initial state is studied as well. The short-time dynamics exhibits initial slips on the scale of the cutoff frequency of the bath modes. Moreover, whereas for a single bath decay of coherence depends crucially on the chosen initial state, for a symmetric coupling to two baths this dependence vanishes.
\end{abstract}

DOI: 10.1103/PhysRevB.88.205122

PACS number(s): 03.65.Yz, 03.67.Lx

\section{INTRODUCTION}

Under the notations of frustration of decoherence or quantum frustration, effects are subsumed which are ascribed to the competition and mutual cancellation of two environments, which couple to noncommuting observables of a central system. The notion was coined in Ref. 1, and the effect has since then been studied, in a variety of systems, such as a two-level system (TLS) coupled to two oscillator baths ${ }^{1-3}$ or to two spin baths, ${ }^{4}$ a harmonic oscillator coupled to two oscillator baths $^{5-8}$ in spin lattices ${ }^{9}$ or Josephson networks. ${ }^{10}$ Most notably, it was proposed as a cooling mechanism. ${ }^{11}$ The relation to Kondo physics was already pointed out in Ref. 1. Certain phenomena occurring in the two-channel Kondo model or in the Bose-Fermi Kondo model can actually be interpreted in terms of quantum frustration. ${ }^{12,13}$

In the model originally studied in Refs. 1 and 2, a TLS with energy gap $\Delta$ couples linearly with its two transversal components to two independent baths. It will be called 2BTLS in the following. The strength of the Ohmic coupling is measured by two quantities $\gamma_{3}^{(1)}$ and $\gamma_{2}^{(2)}$ (assuming a magnetic field in the $x$ direction, bath 1 couples to the $z$ component and bath 2 to the $y$ component). One remarkable result of Ref. 1 includes the renormalization group (RNG) equations

$$
\begin{aligned}
\frac{d \gamma_{3}^{(1)}}{d l} & =-2 \gamma_{3}^{(1)} \gamma_{2}^{(2)}-\gamma_{3}^{(1)} h^{2}, \\
\frac{d \gamma_{2}^{(2)}}{d l} & =-2 \gamma_{3}^{(1)} \gamma_{2}^{(2)}-\gamma_{2}^{(2)} h^{2}, \\
\frac{d h}{d l} & =\left(1-\gamma_{3}^{(1)}-\gamma_{2}^{(2)}\right) h,
\end{aligned}
$$

where $d l=-d \ln \omega_{c}$ is the differential of the flow parameter, $h=\Delta / \omega_{c}$, and $\omega_{c}$ is the cutoff frequency of the bath modes. If either $\gamma_{3}^{(1)}$ or $\gamma_{2}^{(2)}$ is zero, the RNG equations of the single-bath spin-boson model ${ }^{14,15}$ are recovered which predict a Kosterlitz-Thouless (KT) phase transition for $\gamma=1$. For $\gamma_{3}^{(1)}=\gamma_{2}^{(2)}$, the renormalization flow is different: $h$ scales always to infinity, i.e., a phase transition never occurs, not even for arbitrary strong coupling. This is by now one of the most striking signatures of quantum frustration.
However, the question as to whether for large couplings the delocalized phase at symmetric coupling is stable against asymmetries remains unanswered by the above RNG equations. They do not yield any estimate for the renormalized energy gap $\Delta_{r}$, respectively, Kondo temperature in the delocalized phase.

The body of publications mentioned above focuses on thermal equilibrium. But, the question as to whether or not quantum coherence of a nonequilibrium initial state is protected by quantum frustration is crucial for possible applications. Time evolution of a spin in nonequilibrium can be more complicated than exponential decay predicted by Bloch equations. ${ }^{16}$ In particular, an initially decoupled central system might on a very short-time scale, called quantum Zeno time, incur initial slips. This happens for instance to the dissipative harmonic oscillator. ${ }^{17}$ It was shown in Refs. 5 and 6 that for the harmonic oscillator at short times decoherence is enhanced by a second bath and only later effects of quantum frustration occur.

We address the above questions for the 2BTLS using the method of Hamiltonian flow equations. Flow equations were introduced in the early 1990s by Głazek and Wilson ${ }^{18}$ and at about the same time by Wegner. ${ }^{19}$ The method rests upon a continuous diagonalization of the Hamiltonian. It was applied to the single-bath spin-boson model in Refs. 21-24. In particular, it proved to yield good results for the renormalized energy gap $\Delta_{r}$. More details can be found in Ref. 20 .

In this work, a generalization of Eq. (1) is derived analytically, which embraces any kind of coupling to two baths. Numerically, $\Delta_{r}$ is calculated as a function of an asymmetry angle, called $\theta$, which varies from zero (single bath) to $\pi / 4$ (completely symmetric). Whereas for weak coupling there is little dependence on the asymmetry angle, as the coupling becomes stronger the dependence on the asymmetry becomes more and more important. A symmetric coupling protects the gap and prevents the KT phase transition. Identifying the critical angle allows us to plot a phase diagram in the $\gamma_{3}^{(1)}-\gamma_{2}^{(2)}$ plane, where the localized and the delocalized phases are separated by a critical line.

Using techniques developed recently, ${ }^{25,26}$ the question is addressed as to whether decoherence of a nonequilibrium initial state is protected by a second bath. The answer can not 
be given without a careful distinction about what is meant by quantum decoherence. In a folkloristic definition, decoherence is the decay of the off-diagonal elements of the system's reduced density matrix $\rho_{S}$ in some pointer basis and relaxation the decay of the diagonal elements. For a two-level system, both processes are obviously not independent and it is therefore not easy to distinguish them.

Whereas for large times $t \gg \omega_{c}^{-1}$ relaxation and decoherence of a nonequilibrium initial state are well approximated by Bloch equations, short-time dynamics turns out to be rather complicated. The expectation values of the spin operators incur initial slips and subsequent oscillations on a time scale of the cutoff frequency $\omega_{c}$. We will argue that the oscillations are due to the sharp cutoff function and disappear if a smooth cutoff function is used. We will show that the magnitude of the slip is typically increased by switching on a second bath. Moreover, for a single bath the magnitude of the slip depends crucially on the chosen initial state. This dependence vanishes for a symmetric coupling to two baths due to a $U(1)$ symmetry of the Hamiltonian.

The paper is organized as follows: In Sec. II, we set up the model and derive the flow equations. In Sec. III, the renormalized tunnel matrix element and equilibrium quantities are calculated. In Sec. IV, the nonequilibrium dynamics is considered.

\section{FLOW EQUATIONS FOR THE 2BTLS}

\section{A. Derivation of the flow equations}

The Hamiltonian of the 2BTLS is given by $(\hbar=1)$

$$
\begin{aligned}
H^{(0)}= & H_{0}+H_{I}^{(0)}, \\
H_{0}= & -\Delta S_{1}+\sum_{n=1}^{2} \sum_{k} \omega_{k}^{(n)} a_{n, k}^{\dagger} a_{n, k}, \\
H_{\mathrm{I}}^{(0)}= & S_{3} \otimes \sum_{k} \lambda_{3, k}^{(1)}\left(a_{1, k}+a_{1, k}^{\dagger}\right) \\
& +i S_{2} \otimes \sum_{k} \lambda_{2, k}^{(2)}\left(a_{2, k}-a_{2, k}^{\dagger}\right),
\end{aligned}
$$

where $S_{i}$ are spin- $\frac{1}{2}$ matrices and $a_{n, k}$ are bosonic annihilation operators $\left[a_{n, k}, a_{m, k^{\prime}}^{\dagger}\right]=\delta_{k k^{\prime}} \delta_{n m}$ and $\left[a_{n, k}, a_{m, k^{\prime}}\right]=\left[a_{n, k}^{\dagger}, a_{m, k^{\prime}}^{\dagger}\right]$ $=0$. We will also use $S_{0}=\frac{1}{2} \mathbb{1}_{2}$. The sum runs over the $N$ bath modes, where $N$ is assumed a large number such that the spectral functions

$$
\begin{aligned}
& J_{3}^{(1)}(\omega)=\sum_{k}\left(\lambda_{3, k}^{(1)}\right)^{2} \delta\left(\omega-\omega_{k}^{(1)}\right), \\
& J_{2}^{(2)}(\omega)=\sum_{l}\left(\lambda_{2, l}^{(2)}\right)^{2} \delta\left(\omega-\omega_{l}^{(2)}\right)
\end{aligned}
$$

of both baths are smooth functions. They obey an Ohmic power law for small frequencies $J_{3}^{(1)}(\omega)=2 \gamma_{3}^{(1)} \omega$ and $J_{2}^{(2)}(\omega)=$ $2 \gamma_{2}^{(2)} \omega$ and are regularized by a cutoff $\omega_{c} \gg \Delta$. For simplicity, we assume here and in the following the cutoff and the number of bath modes to be the same for both baths.

The Hamiltonian is band diagonalized by a unitary transformation $^{18-20}$ which depends continuously on a flow parameter $B$. A one-parameter family of unitarily equivalent
Hamiltonians $H^{(0)}(B)=U(B) H^{(0)} U^{\dagger}(B)$ is considered, where $H^{(0)}(B)$ obeys the flow equation

$$
\frac{d}{d B} H^{(0)}(B)=\left[\eta^{(0)}(B), H^{(0)}(B)\right] .
$$

The anti-Hermitian operator $\eta^{(0)}=\left(\frac{d}{d B} U\right) U^{\dagger}$ is the generator of the transformation. If $\eta^{(0)}$ is chosen as the commutator $\eta^{(0)}=$ $\left[H_{0}(B), H_{I}^{(0)}(B)\right]$ and if $H_{0}$ is nondegenerate, the Hamiltonian $H^{(0)}(\infty)$ becomes diagonal. ${ }^{27}$ To see this, it is sufficient to consider the derivative of $F=\operatorname{tr}\left(H_{I}^{(0)}\right)^{2}$ with respect to the flow parameter $B$ :

$$
\frac{d}{d B} F(B)=-2 \sum_{k, k^{\prime}}\left(\varepsilon_{k}-\varepsilon_{k^{\prime}}\right)^{2} H_{k k^{\prime}}^{(0)},
$$

where $\varepsilon_{k}$ are eigenvalues of $H_{0}$. For nondegenerate $H_{0}$, the right-hand side of (5) is positive definite and $\lim _{B \rightarrow \infty} F(B)=$ 0 . For large but finite $B$, the Hamiltonian becomes band diagonal with bandwidth $\sim \sqrt{B}$. The canonical generator reads as

$$
\begin{aligned}
\eta^{(0)}= & S_{3} \otimes \sum_{k}\left\{\Delta \lambda_{2, k}^{(2)}\left(a_{2, k}-a_{2, k}^{\dagger}\right)-\omega_{k}^{(1)} \lambda_{3, k}^{(1)}\left(a_{1, k}-a_{1, k}^{\dagger}\right)\right\} \\
& +i S_{2} \otimes \sum_{k}\left\{\Delta \lambda_{3, k}^{(1)}\left(a_{1, k}+a_{1, k}^{\dagger}\right)-\omega_{k}^{(2)} \lambda_{2, k}^{(2)}\left(a_{2, k}-a_{2, k}^{\dagger}\right)\right\} .
\end{aligned}
$$

The commutator of $\eta^{(0)}$ with $H^{(0)}$ generates interaction terms not present in $H^{(0)}$. They must formally be included in a more general Hamiltonian $H=H^{(0)}+H^{(1)}$ and likewise in a new generator $\eta=\left[H_{0}, H\right]$. In the resulting double commutator $\left[\left[H_{0}, H\right], H\right]$, all normal-ordered products of more than two creation or annihilation operators are neglected. Thereby a closed set of differential equations for the parameters of $H$ is obtained.

In order to write the form-invariant Hamiltonian $H$ and the generator $\eta$ in a compact form, it is useful to arrange the creation and annihilation operators in a $4 N$ vector $\vec{A}^{T}=$ $\left[\vec{a}_{1}^{T},\left(\vec{a}_{1}^{\dagger}\right)^{T},{\overrightarrow{a_{2}}}^{T},\left({\overrightarrow{a_{2}}}^{\dagger}\right)^{T}\right]$, where $\vec{a}_{n}^{T}=\left(a_{n, 1}, \ldots, a_{n, N}\right),\left(\vec{a}_{n}^{\dagger}\right)^{T}=$ $\left(a_{n, 1}^{\dagger}, \ldots, a_{n, N}^{\dagger}\right), n=1,2$. It turns out useful as well to introduce coupling constants $\lambda_{ \pm, k}^{(n)} \equiv \lambda_{3, k}^{(n)} \pm \lambda_{2, k}^{(n)}$ and arrange them in a $4 N$ vector $\vec{\Lambda}=\left(\vec{\lambda}_{+}^{(1) T}, \vec{\lambda}_{-}^{(1) T}, \vec{\lambda}_{+}^{(2) T}, \vec{\lambda}_{-}^{(2) T}\right)$, where $\vec{\lambda}_{ \pm}^{(n) T}=\left(\lambda_{ \pm, 1}^{(n)}, \ldots, \lambda_{ \pm, N}^{(n)}\right), n=1,2$. Moreover, $S_{ \pm}=$ $\left(S_{3} \pm i S_{2}\right) / 2$. Then,

$$
H_{\mathrm{I}}=S_{+} \otimes \vec{\Lambda}^{T} \vec{A}+S_{-} \otimes \vec{A}^{\dagger} \vec{\Lambda}+S_{1} \otimes: \vec{A}^{\dagger} T \vec{A}: .
$$

The symbol $: a b:$ denotes normal ordering with respect to a thermal expectation value. The $4 N \times 4 N$ matrix $T$ has the following block structure:

$$
\begin{aligned}
T & =\left(\begin{array}{llll}
s_{11} & t_{11} & s_{12} & t_{12} \\
t_{11} & s_{11} & t_{12} & s_{12} \\
s_{12}^{T} & t_{12}^{T} & s_{22} & t_{22} \\
t_{12}^{T} & s_{12}^{T} & t_{22} & s_{22}
\end{array}\right), \quad t_{i i}=t_{i i}^{T}, \\
s_{i i} & =s_{i i}^{T}, \quad t_{i j}, s_{i j} \in \mathbb{R} .
\end{aligned}
$$

Note the invariance of $T$ under the unitary automorphism $T \rightarrow \Sigma_{x}^{-1} T \Sigma_{x}$, where $\Sigma_{x}=\mathbb{1}_{2} \otimes \sigma_{x} \otimes \mathbb{1}_{N}$ and $\sigma_{x}=\left[\begin{array}{ll}0 & 1 \\ 1 & 0\end{array}\right]$ is 
a Pauli matrix. Likewise, we define $\Sigma_{z}$. The generator reads as

$$
\begin{aligned}
\eta= & S_{+} \otimes \vec{\Lambda}^{T}(\Delta-\Omega) \vec{A}-S_{-} \otimes \vec{A}^{\dagger}(\Delta-\Omega) \vec{\Lambda} \\
& +S_{1} \otimes: \vec{A}^{\dagger}[\Omega, T] \vec{A}:
\end{aligned}
$$

where $\Omega=\operatorname{diag}\left(\omega^{(1)},-\omega^{(1)}, \omega^{(2)},-\omega^{(2)}\right)$ and $\omega^{(n)}=$ $\operatorname{diag}\left(\omega_{1}^{(n)}, \ldots, \omega_{N}^{(n)}\right), n=1,2$. In former treatments of the spinboson model with a single bath ${ }^{21,23}$ within the flow-equation approach, a formally simpler generator was used instead of the canonical one $\eta=\left[H_{0}, H\right]$. This reduced the number of differential equations to be solved. The different generators were contrasted in Ref. 28. In general, there seems to exist by now no other guideline to improve the canonical generator than an educated guess or physical intuition. Thus, for the present problem we stick to the canonical one.

The commutator $[\eta, H]$ is calculated straightforwardly and a set of nonlinear coupled ordinary differential equations (ODE's) is obtained for the tunneling matrix element $\Delta$, the couplings $\vec{\Lambda}$ and for the matrix elements of $T$. They read as

$$
\begin{aligned}
\frac{d \Delta(B)}{d B}= & \frac{1}{2} \vec{\Lambda}^{T}(\Delta-\Omega) \operatorname{coth}\left(\frac{\beta|\Omega|}{2}\right) \vec{\Lambda}, \\
\frac{d \vec{\Lambda}(B)}{d B}= & -(\Delta-\Omega)^{2} \vec{\Lambda}+\{T(\Delta-\Omega) \\
& +[\Omega, T]\} \operatorname{coth}\left(\frac{\beta|\Omega|}{2}\right) \vec{\Lambda}, \\
\frac{d T(B)}{d B}= & -[\Omega,[\Omega, T]]-\frac{1}{2} \vec{\Lambda}(\Delta-\Omega) \vec{\Lambda}^{T} \\
& -\frac{1}{2} \Sigma_{x} \vec{\Lambda}(\Delta-\Omega) \vec{\Lambda}^{T} \Sigma_{x} .
\end{aligned}
$$

The equations (10) form a set of $1+4 N+2 N(4 N+1)$ firstorder nonlinear differential equations which must be solved numerically. Before we do so, we show in the next section how they reduce to the RNG equations (1). The reader, who is not interested in this point, might skip this section and continue directly with Sec. III.

\section{B. Derivation of the RNG equations from Eq. (10)}

We derive the RNG equations (1) from Eq. (10) for an Ohmic bath in the low-frequency limit. We limit ourselves to zero temperature. The differential equations for the entries of $T$ are of the type

$$
\frac{d f(B)}{d B}=\omega f(B)+g(B), \quad \omega \in \mathbb{R}
$$

which can be solved exactly

$$
f(B)=f(0) e^{\omega B}+\int_{0}^{B} d B^{\prime} e^{\omega\left(B-B^{\prime}\right)} g\left(B^{\prime}\right) .
$$

This might be plugged into the flow equation for $\vec{\Lambda}$. It suffices to evaluate these equations for small frequencies. Using the definitions of the spectral functions (3) and

$$
\begin{aligned}
\sum_{k} \lambda_{j, k}^{(n)}(B) \lambda_{j^{\prime}, k}^{(n)}\left(B^{\prime}\right) \delta\left(\omega-\omega_{k}^{(n)}\right) & =2 \sqrt{\gamma_{j}^{(n)} \gamma_{j^{\prime}}^{(n)}} \omega, \\
\forall j, j^{\prime} & \in\{2,3\}
\end{aligned}
$$

an integrodifferential equation for the coupling constants is acquired:

$$
\begin{aligned}
\frac{d \gamma_{3}^{(n)}(B)}{d B}= & -2 \Delta(B)^{2} \gamma_{3}^{(n)}(B)-2 \int_{0}^{B} d B^{\prime} \sqrt{\gamma_{3}^{(n)}(B) \gamma_{3}^{(n)}\left(B^{\prime}\right) \omega_{c}^{2}} \\
& \times \int_{0}^{1} d x e^{-x \omega_{c}^{2}\left(B-B^{\prime}\right)} \sum_{m=1}^{2}\left(\Delta(B) \sqrt{\gamma_{3}^{(m)}(B)}\right. \\
& -2 \omega_{c} \sqrt{\left.\gamma_{2}^{(m)}(B) x\right)} \\
& \times\left(2 \Delta\left(B^{\prime}\right) \sqrt{\gamma_{3}^{(m)}\left(B^{\prime}\right)}-\omega_{c} \sqrt{\left.\gamma_{2}^{(m)}\left(B^{\prime}\right) x\right)} .\right.
\end{aligned}
$$

The corresponding equation for $\gamma_{2}^{(n)}$ is obtained from Eq. (14) by interchanging the indices 2 and 3 everywhere. This equation permits a perturbative expansion in $h=\Delta / \omega_{c}$. Keeping only the highest-order term in the integral, Eq. (14) reduces to

$$
\begin{aligned}
\frac{d \gamma_{3}^{(n)}(B)}{d B}= & -2 \omega_{c}^{2} h^{2}(B) \gamma_{3}^{(n)}(B) \\
& -4 \int_{0}^{B} d B^{\prime} \sqrt{\gamma_{3}^{(n)}(B) \gamma_{3}^{(n)}\left(B^{\prime}\right)} \omega_{c}^{4} \\
& \times \int_{0}^{1} d x x e^{-x \omega_{c}^{2}\left(B-B^{\prime}\right)} \sum_{m=1}^{2} \sqrt{\gamma_{2}^{(m)}(B) \gamma_{2}^{(m)}\left(B^{\prime}\right) .}
\end{aligned}
$$

In the limit $\omega_{c} \rightarrow \infty$, the $B^{\prime}$ integration becomes $\delta$ like for almost all $x \in[0,1]$ and we arrive at

$$
\frac{d \gamma_{3}^{(n)}(B)}{d B}=-2 \omega_{c}^{2} h^{2}(B) \gamma_{3}^{(n)}(B)-4 \gamma_{3}^{(n)}(B) \omega_{c}^{2} \sum_{m=1}^{2} \gamma_{2}^{(m)}(B)
$$

and likewise for $\gamma_{2}^{(n)}(B)$. To make contact with the RNG equations, we use the relation ${ }^{20}$

$$
\omega_{c}=\frac{1}{2 \sqrt{B}}=e^{-l}
$$

and obtain

$$
\begin{aligned}
& \frac{d \gamma_{2}^{(n)}(l)}{d l}=h^{2} \gamma_{2}^{(n)}-2 \gamma_{2}^{(n)} \sum_{m=1}^{2} \gamma_{3}^{(m)}, \\
& \frac{d \gamma_{3}^{(n)}(l)}{d l}=h^{2} \gamma_{3}^{(n)}-2 \gamma_{3}^{(n)} \sum_{m=1}^{2} \gamma_{2}^{(m)}, \quad n=1,2 .
\end{aligned}
$$

On the other hand, a differential equation for $h$ is straightforwardly derived from Eq. (10):

$$
\frac{d h(l)}{d l}=\left(1-\sum_{j=2,3} \sum_{m=1}^{2} \gamma_{j}^{(m)}\right) h .
$$

Equations (18) and (19) correspond to the perturbative renormalization group equations for arbitrary couplings $\gamma_{2}^{(n)}$ and 

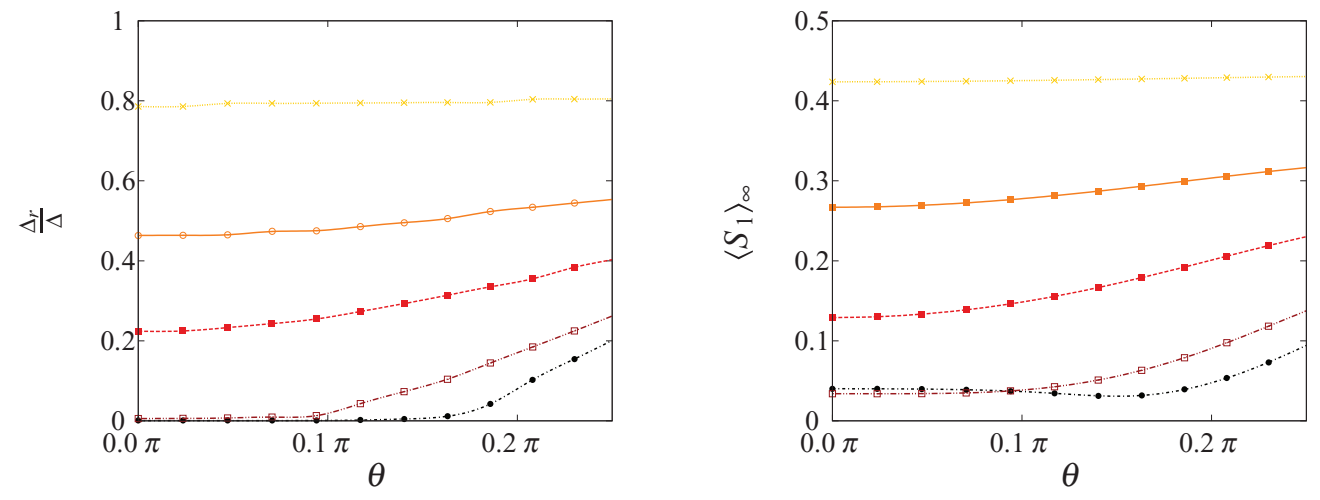

FIG. 1. (Color online) Left: Plot of the renormalized tunneling matrix element $\Delta_{r}$ as a function of the angle $\theta$ defined in the main text, the total coupling strength is $\gamma_{\text {tot }}=0.1$ (crosses, online yellow), $\gamma_{\text {tot }}=0.3$ (empty circles, online orange), $\gamma_{\text {tot }}=0.5$ (filled boxes, online red), $\gamma_{\mathrm{tot}}=0.8$ (empty boxes, online dark red), and $\gamma_{\mathrm{tot}}=1$ (filled circles, full black line). The cutoff frequency is $\omega_{c}=10 \Delta$. The number of bath modes is $N=1000$. Right: the same for the equilibrium expectation value $\left\langle S_{1}\right\rangle_{\infty}$. $\omega_{c}=10 \Delta, N=400$.

$\gamma_{3}^{(n)}, n=1,2$. We do not analyze them further here, but only mention that the result of Novais et al. ${ }^{2}$ stated in Eq. (1) is obtained by setting $\gamma_{2}^{(1)}$ and $\gamma_{3}^{(2)}$ to zero. However, it must be pointed out that the same equations are obtained for $\gamma_{3}^{(1)}$ and $\gamma_{2}^{(1)}$, if $\gamma_{3}^{(2)}$ and $\gamma_{2}^{(2)}$ are set to zero, i.e., in the absence of the second bath.

\section{EQUILIBRIUM PROPERTIES}

\section{A. Renormalized tunneling matrix element}

The flow equations (10) are numerically integrated up to a large end value $B_{\max }$, where $1 / \sqrt{B_{\max }}$ is of order of the mean level spacing of the unperturbed Hamiltonian $H_{0}$. The oscillator modes are uniformly distributed in the interval $\left[0, \omega_{c}\right]$ such that the mean level spacing is constant.

An adaptive step-size fourth-order Runge-Kutta algorithm has proved to be a reliable solver. Most entries of $\vec{\Lambda}$ and of $T$ become exponentially small for large flow parameter and the Hamiltonian becomes diagonal

$$
H(\infty)=-\Delta_{r} S_{1}+\frac{1}{2}: \vec{A}^{\dagger}|\Omega| \vec{A}:+H_{\text {res }}
$$

with a finite renormalized tunneling matrix element $\Delta_{r} \equiv$ $\Delta(\infty)$. Not all entries of $\vec{\Lambda}$ and of $T$ decay exponentially for large $B$. From the flow equations (10) it is seen that the coupling coefficients $\lambda_{+, k}^{(n)}$ for frequencies close to the renormalized tunneling matrix element decay most slowly. On the other hand, the diagonal entries of $T$ do not decay at all, leading to an effective coupling of the bath modes to $S_{1}$ in the renormalized Hamiltonian

$$
H_{\text {res }}=S_{1} \otimes \sum_{n=1}^{2} \sum_{k} s_{n n, k k}(\infty) a_{n, k}^{\dagger} a_{n, k} .
$$

Although this term, being diagonal, causes no additional difficulties, for practical purposes it can be neglected since the residual matrix elements $s_{11, k k}, s_{22, k k}$ are usually much smaller than the mean level spacing of the bath modes.

In Fig. 1, the renormalized energy gap of the two-level system is plotted for a fixed overall coupling $\gamma_{\text {tot }} \equiv \gamma_{3}^{(1)}+\gamma_{2}^{(2)}$ as a function of the relative angle $\theta \equiv \arctan \left(\sqrt{\gamma_{2}^{(2)} / \gamma_{3}^{(1)}}\right)$ which varies from zero (single bath) to $\pi / 4$ (symmetric coupling). Whereas for small overall coupling the renormalized energy gap $\Delta_{r}$ is almost independent of $\theta$, for increasing coupling strength the gap is protected by a symmetric coupling. For $\gamma_{\text {tot }}=1$, the energy gap renormalizes to zero for $\theta=0$ but remains finite for $\theta=\pi / 4$.

If $\gamma_{\text {tot }}$ is increased even further, the energy gap $\Delta(B)$ crosses zero for some large value of $B$ and decays afterwards very slowly in an oscillatory fashion to zero. This happens for angles smaller than some critical angle, indicating the onset of the strong coupling regime, respectively, of the KT phase transition. It is expected that the flow equations, being generically perturbative, become less exact for stronger coupling. However, for $\theta=0$ the critical value $\gamma=1$ was obtained analytically and with good precision numerically. ${ }^{21}$ Therefore, it is well justified to assume that the flow equations yield good estimates for the critical $\gamma_{\text {tot }}$ for $\theta \neq 0$ as well.

In Fig. 2, the critical line is plotted in the $\gamma_{\text {tot }}-\theta$ plane, which separates the localized from the delocalized phase. It is seen that it crosses the $x$ axis at some value smaller than one. This offset is due to the finite number of bath modes and of the finite cutoff frequency. This error can be improved systematically by increasing the number of bath modes and simultaneously

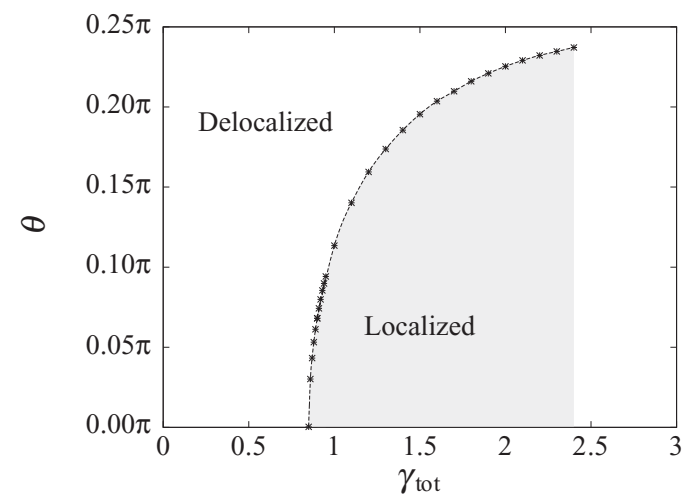

FIG. 2. Phase diagram in the $\gamma_{\mathrm{tot}^{-}}-\theta$ plane. The line indicates the critical asymmetry angle, which separates the localized from the delocalized phase. The critical angle was determined for $N=800$ bath modes. 
increasing the end point of the flow $B_{\max }$. For values of $\gamma_{\text {tot }}$ larger than some value $\gamma_{\text {tot }} \approx 2.5$, the flow becomes unstable.

\section{B. Equilibrium correlation functions}

In order to calculate equilibrium expectation values with respect to the transformed Hamiltonian $H(\infty)$, the corresponding operators have to transform as well. Complex $4 N$ vectors $\vec{\chi}$ and $\vec{\zeta}_{0,1}$ are introduced and the spin operators are expanded as

$$
\begin{aligned}
& S_{1}=h_{0} S_{0}+h_{1} S_{1}+S_{+} \otimes \vec{\chi}^{\dagger} \vec{A}+S_{-} \otimes \vec{A}^{\dagger} \vec{\chi}, \\
& S_{+}=h_{+} S_{+}+h_{-} S_{-}+S_{0} \otimes \vec{\zeta}_{0}^{\dagger} \vec{A}+S_{1} \otimes \vec{\zeta}_{1}^{\dagger} \vec{A}, \\
& S_{-}=h_{-}^{*} S_{+}+h_{+}^{*} S_{-}+S_{0} \otimes \vec{A}^{\dagger} \vec{\zeta}_{0}+S_{1} \otimes \vec{A}^{\dagger} \vec{\zeta}_{1} .
\end{aligned}
$$

The flow equations for $h_{0}, h_{1}, h_{ \pm}$and for $\vec{\chi}, \vec{\zeta}_{0,1}$ are obtained by calculating the commutator $\left[\eta, S_{i}\right]$. The equations are closed by neglecting all normal-ordered operator products with two or more annihilation or creation operators. They are stated in Appendix B. The equilibrium density matrix with respect to the renormalized free Hamiltonian (20) is just

$$
\rho_{\beta}=\left[S_{0}+\tanh \left(\frac{\Delta_{r} \beta}{2}\right) S_{1}\right] \otimes \rho_{\beta}^{(1)} \otimes \rho_{\beta}^{(2)} .
$$

Here, $\rho_{\beta}^{(n)}=\prod_{k} \exp \left(-\beta \omega_{k}^{(n)} a_{n, k}^{\dagger} a_{n, k}\right) / Z^{(n)}(\beta)$ is the thermal density matrix of the two free environments. Thus, once the equations are numerically solved, an arbitrary equilibrium expectation value of the spin operators is readily calculated. As an example, we consider the one-sided Fourier transform

$$
\chi_{z z}(\omega)=-i \int_{0}^{\infty} \frac{d t}{2 \pi} e^{i \omega t}\left\langle\left[S_{3}(0), S_{3}(t)\right]\right\rangle_{\beta}
$$

of the correlator $\left\langle\left[S_{3}(0), S_{3}(t)\right]\right\rangle_{\beta}$ which was investigated in Ref. 2. At zero temperature $\beta=\infty$, its imaginary part $\chi_{z z}^{\prime \prime}$ is given by

$$
\begin{aligned}
\chi_{z z}^{\prime \prime}(\omega) \propto & \left(h_{+}+h_{-}\right)^{2} \delta\left(\omega-\Delta_{r}\right) \\
& +\left(\vec{\zeta}_{0}+\vec{\zeta}_{1}\right)^{\dagger}\left(1+\Sigma_{x}\right) \delta(\omega-|\Omega|)\left(\vec{\zeta}_{0}+\vec{\zeta}_{1}\right) .
\end{aligned}
$$

As a second example, we consider the equilibrium expectation value

$$
\left\langle S_{1}\right\rangle_{\beta}=\frac{h_{0}}{2}+\frac{h_{1}}{2} \tanh \left(\frac{\Delta_{r} \beta}{2}\right) .
$$

It is plotted in the right picture of Fig. 1 for zero temperature and for different angles $\theta$ as defined before. Since the calculation is numerically more expensive than that of the energy gap, the number of bath modes is $N=400$. For small and intermediate coupling, it behaves qualitatively similar to the renormalized two-level energy gap $\Delta_{r}$. For strong coupling $\gamma_{\text {tot }} \approx 1$ it is seen that $\left\langle S_{1}\right\rangle_{\infty}$ does not scale to zero for $\theta=0$ as expected, indicating that the flow equations lose accuracy in the strong coupling regime.

Before we discuss the numerical results for the equilibrium correlation functions, an explanatory remark is in order. A careful treatment of equilibrium correlation functions within the flow-equation approach requires high sophistication. For frequencies close to the renormalized tunnel matrix element $\Delta_{r}$, the flow converges only very slowly with $B_{\max }$, the end point of the numerical integration of the flow. Since the

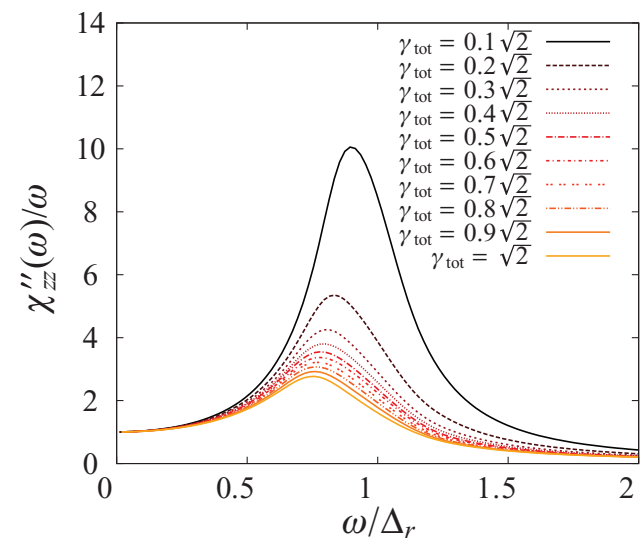

FIG. 3. (Color online) Plot of the transverse susceptibility $\chi_{z z}^{\prime \prime}(\omega) / \omega$ in the $z$ direction for symmetric coupling $\gamma_{3}^{(1)}=\gamma_{2}^{(2)}$ and for 10 different values of $\gamma_{\text {tot }}=\sqrt{2} \times 0.1 n, 1 \leqslant n \leqslant 10$, from top to bottom (online color: from dark colored to light colored). The number of bath modes is $400, \Delta / \omega_{c}=\frac{1}{10}$.

end point of the integration is itself limited by the density of the bath modes, an accurate resolution would require an out-of-scale number of bath modes. As a consequence of this numerical limitation, the equilibrium correlation functions have a two-peak structure: one broad maximum at a value smaller than $\Delta_{r}$ and a second sharp peak right at $\Delta_{r}$, which is clearly unphysical. The problem can be overcome by employing constants of motion under the flow. This was done in Ref. 23 for the one-bath spin-boson model. The result is a smooth curve with a single peak. But, such constants of motion under the flow are not always easy to identify.

We refrain from this procedure and show the curves for $\chi_{z z}^{\prime \prime}(\omega)$ obtained by fitting the numerical data with smoothing splines using an extremely high fidelity factor (of order $10^{8}$ ) everywhere but around $\Delta_{r}$, where it is quartically suppressed. The numerics were performed with a sharp cutoff frequency of $\omega_{c}$ and with 400 oscillator modes, from which 300 were condensed into the interval $[0,2 \Delta]$ in order to get a good resolution in the region around the resonance.

In Fig. 3, the correlation function $\chi_{z z}^{\prime \prime}(\omega)$ is plotted for equal coupling strength to both baths $(\theta=\pi / 4)$ and with an overall coupling strength $\gamma_{\text {tot }}$ varying between $0.1 \times \sqrt{2}$ and $\sqrt{2}$. The curve corresponds to Fig. 4 in Ref. 2 and is qualitatively similar. As the coupling strength increases, the resonance peak becomes smaller and smaller but never disappears. The maximum of the resonance peak is systematically below $\Delta_{r}$. This is a difference to Fig. 4 in Ref. 2 where the maximum seems to be always right at the renormalized tunnel matrix element.

In Fig. 4, the correlation function $\chi_{z z}^{\prime \prime}(\omega)$ is plotted for fixed overall coupling strength $\gamma_{\text {tot }}$ and for different symmetry angles $\theta$. The resonance peak in the symmetric case $(\theta=\pi / 4)$ is largely enhanced as compared to the highly asymmetric case $(\theta=0.1 \pi)$. However, the reason for this is rather trivial. In the highly asymmetric case, the coupling to the $z$ component is largest, whereas there is no coupling to the $y$ component. In the symmetric case, the coupling to the $z$ component is reduced, which is reflected by the enhanced resonance peak of $\chi_{z z}^{\prime \prime}$. However, the coupling to the $y$ component is larger, 


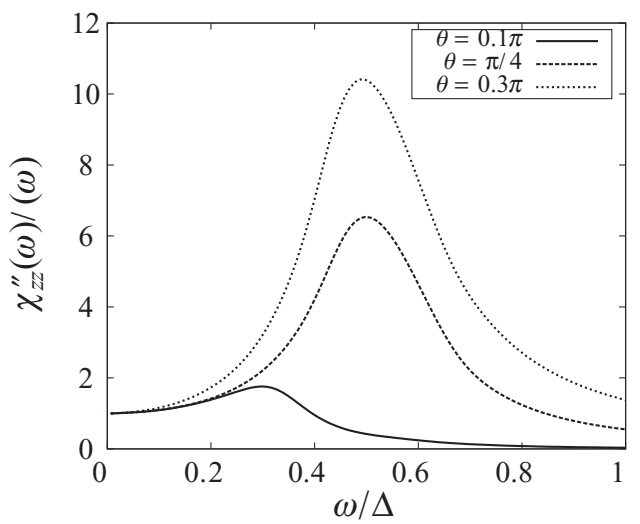

FIG. 4. Plot of the transverse susceptibility $\chi_{z z}^{\prime \prime}(\omega) / \omega$ in the $z$ direction for three different angles $\theta=0.1 \pi$ (full line), $\theta=\pi / 4$ (dashed line), and $\theta=0.3 \pi$ (dotted line) for overall coupling strength $\gamma_{\text {tot }}=0.3$. The number of bath modes is $N=400, \omega_{c} / \Delta=10$.

which yields a reduced resonance peak of $\chi_{y y}^{\prime \prime}$ (not shown here). If we write $\chi_{z z}^{\prime \prime}(\omega, \theta)$ as a function of the relative angle $\theta$, then the obvious relation $\chi_{z z}^{\prime \prime}(\omega, \theta)=\chi_{y y}^{\prime \prime}(\omega, \pi / 2-\theta)$ holds. Thus, an enhancement of the resonance peak in the $z$ direction comes necessarily with a decrease in the $y$ direction and vice versa. Indeed, in Fig. 3 the resonance peak of $\chi_{z z}^{\prime \prime}$ is biggest for $\theta=0.3 \pi$ in spite of the asymmetric coupling (for even higher $\theta$ it increases more and more). Note, however, that the location of the maximum of the peak is maximal for symmetric coupling $(\theta=\pi / 4)$.

\section{THERMALIZATION AND DECOHERENCE}

In thermal equilibrium, the mutual energy transfer from the system to the environment and vice versa is zero, warranted by fluctuation dissipation theorems. However, in the process of thermalization the net energy transfer of the system to the environment is positive. Assuming a decoupled initial state, which is fully polarized in some direction perpendicular to the $x$ axis

$$
\rho_{\text {init }}=\left[S_{0}+\cos \left(\theta^{\prime}\right) S_{3}+\sin \left(\theta^{\prime}\right) S_{2}\right] \otimes \rho_{\beta}^{(1)} \otimes \rho_{\beta}^{(2)},
$$

thermalization is characterized by the time evolution of the expectation value of the system's energy $\left\langle H_{S}(t)\right\rangle=-\Delta\left\langle S_{1}(t)\right\rangle$. This quantity is expected to approach its equilibrium value on a certain time scale, the so-called relaxation time, which is usually denoted $T_{1}$.

Decoherence is the creation of entanglement of the system with the environment. It is measured by the decay of the offdiagonal elements of the reduced density matrix of the spin in the $S_{1}$ basis, i.e., by the expectation values $\left\langle S_{ \pm}\right\rangle$. A basisindependent measure for decoherence is the purity $\mathcal{P}(t)=$ $\mathcal{P}_{\|}(t)+\mathcal{P}_{\perp}(t)$, where $\mathcal{P}_{\|}(t)=2 \sum_{n=0}^{1}\left\langle S_{n}(t)\right\rangle^{2}$ and $\mathcal{P}_{\perp}(t)=$ $2 \sum_{n=2}^{3}\left\langle S_{n}(t)\right\rangle^{2}$. Decay of decoherence takes place on a time scale $T_{2}$, called decoherence time, ${ }^{16}$ and we associate it with $\mathcal{P}_{\perp}(t)$. Both decoherence time and relaxation time enter in the definition of purity. We call the two quantities $\mathcal{P}_{\perp}(t)$ and $\mathcal{P}_{\|}(t)$ transverse and parallel purity, respectively. For the initial state (27), $\mathcal{P}_{\perp}(0)=\mathcal{P}_{\|}(0)=\frac{1}{2}$.

\section{A. Bloch equations}

Assuming a decoupled initial state as in Eq. (27), firstorder differential equations for the spin expectation values are straightforwardly derived in second-order perturbation theory:

$$
\begin{aligned}
& \frac{d}{d t}\left\langle S_{1}\right\rangle=-\left(\Gamma_{2}^{(2)}(t)+\Gamma_{3}^{(1)}(t)\right)\left\langle S_{1}\right\rangle-F(t), \\
& \frac{d}{d t}\left\langle S_{2}\right\rangle=\tilde{\Delta}_{3}^{(1)}(t)\left\langle S_{3}\right\rangle-\Gamma_{3}^{(1)}(t)\left\langle S_{2}\right\rangle, \\
& \frac{d}{d t}\left\langle S_{3}\right\rangle=-\tilde{\Delta}_{2}^{(2)}(t)\left\langle S_{2}\right\rangle-\Gamma_{2}^{(2)}(t)\left\langle S_{3}\right\rangle,
\end{aligned}
$$

with the time-dependent coefficients

$$
\begin{aligned}
\Gamma_{n}^{(m)}(t) & =\int_{0}^{t} d t^{\prime} \int_{0}^{\infty} d \omega \cos \left[\Delta\left(t^{\prime}-t\right)\right] \cos \left[\omega\left(t-t^{\prime}\right)\right] J_{n}^{(m)}(\omega) \operatorname{coth}(\omega \beta / 2), \\
\tilde{\Delta}_{n}^{(m)}(t) & =\Delta-\int_{0}^{t} d t^{\prime} \int_{0}^{\infty} d \omega \sin \left[\Delta\left(t^{\prime}-t\right)\right] \cos \left[\omega\left(t-t^{\prime}\right)\right] J_{n}^{(m)}(\omega) \operatorname{coth}(\omega \beta / 2), \\
F(t) & =\int_{0}^{t} d t^{\prime} \int_{0}^{\infty} d \omega \sin \left[\omega\left(t-t^{\prime}\right)\right] \sin \left[\Delta\left(t^{\prime}-t\right)\right]\left(J_{2}^{(2)}(\omega)+J_{3}^{(1)}(\omega)\right) .
\end{aligned}
$$

In the Markov approximation, these coefficients become time independent $\quad \Gamma_{n}^{(m)}(t)=\Gamma_{n}^{(m)}=(\pi / 2) J_{n}^{(m)}(\Delta) \operatorname{coth}(\beta \Delta / 2)$, $F(t)=F=(\pi / 2)\left[J_{2}^{(2)}(\Delta)+J_{3}^{(1)}(\Delta)\right], \quad$ and $\quad \tilde{\Delta}_{n}^{(m)}(t)=$ $\tilde{\Delta}_{n}^{(m)}=\Delta-\Delta \int_{0}^{\omega_{c}} d \omega \operatorname{coth}(\beta \omega / 2) J_{n}^{(m)}(\omega) /\left(\omega^{2}-\Delta^{2}\right)$. Note that for an Ohmic bath and at zero temperature $\tilde{\Delta}_{n}^{(m)}$ has a logarithmic singularity in the cutoff frequency $\omega_{c}$.

From Eqs. (28), the phenomenological Bloch equations are obtained which predict an exponential decay of decoherence and of relaxation. Their solutions are

$$
\begin{aligned}
\left\langle S_{1}(t)\right\rangle= & {\left[\left\langle S_{1}(0)\right\rangle-\left\langle S_{1}\right\rangle_{\mathrm{eq}}\right] e^{-\left(\Gamma_{2}^{(2)}+\Gamma_{3}^{(1)}\right) t}+\left\langle S_{1}\right\rangle_{\mathrm{eq}}, } \\
\left\langle S_{n}(t)\right\rangle= & \frac{\lambda_{+}\left\langle S_{n}(0)\right\rangle+i\left\langle\dot{S}_{n}(0)\right\rangle}{\lambda_{+}-\lambda_{-}} e^{i \lambda_{-} t} \\
& -\frac{\lambda_{-}\left\langle S_{n}(0)\right\rangle+i\left\langle\dot{S}_{n}(0)\right\rangle}{\lambda_{+}-\lambda_{-}} e^{i \lambda_{+} t}
\end{aligned}
$$


where $\lambda_{ \pm}$are the roots of the characteristic polynomial

$$
\chi(\omega)=\omega^{2}-i \omega\left(\Gamma_{2}^{(2)}+\Gamma_{3}^{(1)}\right)+\tilde{\Delta}_{2}^{(2)} \tilde{\Delta}_{3}^{(1)}-\Gamma_{2}^{(2)} \Gamma_{3}^{(1)} .
$$

Thus, decoherence and relaxation time are given by $T_{1}=$ $1 /\left(\Gamma_{2}^{(2)}+\Gamma_{3}^{(1)}\right)$ and $T_{2}=2 T_{1}$. In second-order perturbation theory, the friction coefficients of the two baths add up. No frustration effects occur.

In the Markov approximation, Bloch equations hold beyond perturbation theory with relaxation and decoherence times depending in a more complicated nonperturbative way on the coupling strengths $\gamma_{3}^{(1)}$ and $\gamma_{2}^{(2)}$. Corrections were calculated in Ref. 2. In the regime where the Bloch equations (28) hold, the quantum regression theorem can be invoked and the dynamics of the expectation values is governed by the equilibrium correlation functions.

\section{B. Short-time expansion}

For low temperatures and on the time scale of the inverse cutoff frequency, Bloch equations do not hold. The coefficients in Eq. (28) become time dependent and the simple exponential behavior (30) breaks down. This is seen most directly in a Taylor expansion of the time-evolution operator $U(t)=1-i H t-t^{2} H^{2} / 2+\mathcal{O}\left(t^{3}\right)$. For the initial state (27), it predicts a quadratic behavior of $\left\langle S_{1}\right\rangle=t^{2} / \tau^{2}+\mathcal{O}\left(t^{3}\right)$, with the characteristic time scale

$$
\tau^{-2}=\frac{1}{2} \int_{0}^{\infty} d \omega\left(J_{3}^{(1)}(\omega)+J_{2}^{(2)}(\omega)\right)=\frac{1}{2} \omega_{c}^{2}\left(\gamma_{3}^{(1)}+\gamma_{2}^{(2)}\right) \text {. }
$$

The last equality holds for a sharp cutoff. For $\omega_{c} \gg \Delta$, this time scale is approximately the quantum Zeno time $\tau_{0}$, defined by the energy variance with respect to a given initial state ${ }^{29}$ via

$\tau_{0}^{-2}=\left\langle H^{2}\right\rangle-\langle H\rangle^{2}=\frac{1}{2}\left(\Delta^{2}+\int_{0}^{\infty} d \omega\left(J_{3}^{(1)}(\omega)+J_{2}^{(2)}(\omega)\right)\right)$.

The second equality holds for an initial state characterized by Eq. (27). For the transverse purity, one obtains

$$
\begin{aligned}
2 P_{\perp}(t)= & 1-\frac{2 t^{2}}{\tau^{2}}\left\{\sin ^{2}\left(\theta^{\prime}\right) \cos ^{2}(\theta)\right. \\
& \left.+\sin ^{2}(\theta) \cos ^{2}\left(\theta^{\prime}\right)\right\}+\mathcal{O}\left(t^{3}\right) .
\end{aligned}
$$

The quadratic time dependence vanishes iff $\theta=\theta^{\prime}=0$, i.e., when only one bath is present and the initial spin is aligned into the direction of the spin operator which couples to the oscillator bath. This particular situation might be viewed as a weak form of the quantum Zeno effect, which asserts that a repeated measurement of a state inhibits its decay. ${ }^{30,31}$ Note that for vanishing frequency $\Delta=0$ this initial state does not decay at all.

\section{Flow equations for nonequilibrium states}

In order to monitor the time evolution of the expectation values in the transient regime on a time scale of order of the quantum Zeno time, methods of nonequilibrium real-time thermodynamics must be employed. Real-time quantum evolution is addressed within the flow-equation approach ${ }^{25,26}$ by applying subsequently the unitary transformation $U(B)$ generated by $\eta(B)$ and the time-evolution operator $\exp [-i H(\infty) t]$ on the operator of interest according to the diagram

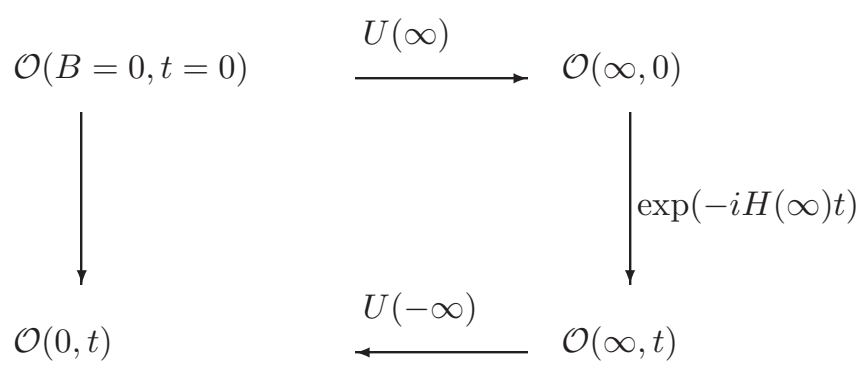

Since time evolution is simple for $B=\infty$, the observables are first transformed into the $B=\infty$ basis and evolve in time and are then transformed back. At time $t$, the Heisenberg operators have been propagated by the diagonalized Hamiltonian (20). This yields new time-dependent expansion coefficients $\tilde{h}_{ \pm}(t)=e^{ \pm i \Delta_{r} t} h_{ \pm}(\infty), \overrightarrow{\tilde{\chi}}(t)=e^{i\left(\Delta_{r}-\Omega\right) t} \vec{\chi}(\infty)$, and $\overrightarrow{\tilde{\zeta}}_{n}(t)=e^{i \Omega t} \vec{\zeta}_{n}(\infty), n=0,1$. The coefficients $h_{0}=\tilde{h}_{0}$ and $h_{1}=\tilde{h}_{1}$ remain constant under time evolution. These coefficients are numerically transformed back, yielding an approximate solution of the Heisenberg equation for the spin operators. The expectation values with respect to the density matrix (27) are

$$
\begin{aligned}
& \left\langle S_{1}(t)\right\rangle=\frac{1}{2} \tilde{h}_{0}(t), \\
& \left\langle S_{2}(t)\right\rangle=\frac{1}{2} \operatorname{Im}\left[\left[\tilde{h}_{+}(t)-\tilde{h}_{-}^{*}(t)\right] e^{i \theta^{\prime}}\right], \\
& \left\langle S_{3}(t)\right\rangle=\frac{1}{2} \operatorname{Re}\left[\left[\tilde{h}_{+}(t)+\tilde{h}_{-}^{*}(t)\right] e^{i \theta^{\prime}}\right] .
\end{aligned}
$$

The calculation is numerically delicate. ${ }^{25,26}$ In order to perform the backward integration, the forward flow of the Hamiltonian must be stored. This is a sizable amount of data of order of one terabyte. The read-in and the read-out slow down the routine. We performed the calculation of $\mathcal{P}_{\perp}$ with 250 uniformly distributed bath modes. Effects of the bath discretization are expected to become important latest on a time scale given by the mean level spacing of the unperturbed Hamiltonian (Heisenberg time). In Appendix $\mathrm{C}$, the relevance of different bath discretizations on $\mathcal{P}_{\perp}$ is investigated in some more detail.

In Fig. 5, the transverse purity is plotted for very short times (left) and for one period of the Rabi oscillation (right) for different four-tuples $\left(\gamma_{\mathrm{tot}}, \omega_{c}, \theta, \theta^{\prime}\right)$. The quadratic approximation (34) fits nicely the numerical results but is valid only for times of order of the inverse cutoff frequency. For $\theta=\theta^{\prime}=0$ (online green curve), the quadratic decay is suppressed and the short-time decay is much slower than in all other cases in agreement with the discussion in Sec. IV B. We call this situation the weak Zeno effect.

For all parameters, the decay occurs in an oscillatory fashion on small and intermediate time scales. As can be seen from the right picture of Fig. 5, the frequency scales with $\omega_{c}$ and the amplitudes with $\gamma_{\text {tot }}$. These oscillations are surprising since purity prima facie is expected to decay smoothly. A revision of the data in Ref. 26 confirmed that these oscillations are implicit there as well. 

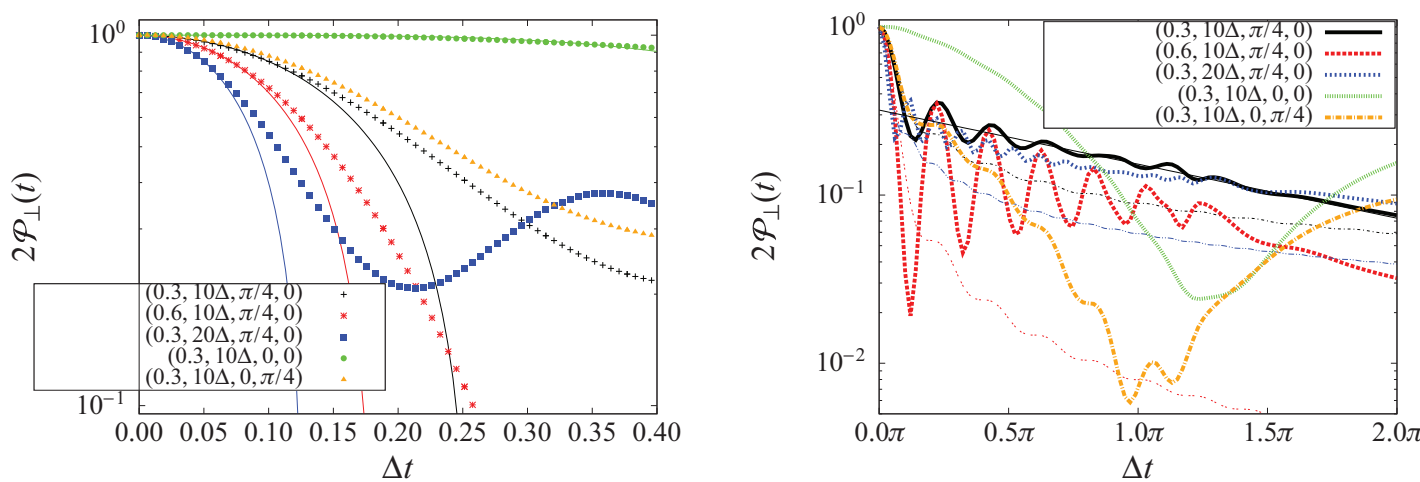

FIG. 5. (Color online) Left: Time evolution on a very short-time scale of the transversal purity characterized by the four-tuples $\left(\gamma_{\mathrm{tot}}, \omega_{c}, \theta, \theta^{\prime}\right)$ for five different cases. The corresponding quadratic approximations (34) are plotted by full lines. Right: The same for one period of the Rabi oscillation. The raw data points are omitted for sake of legibility. The curves of Eq. (36) are plotted in thinner lines for the parameters $\left(\gamma_{\text {tot }}, \omega_{c}\right)=(0.3,10 \Delta)$ (dashed-dotted line, online black), $(0.6,20 \Delta)$ (dashed-dashed line, online red), and $(0.3,20 \Delta)($ dashed-dotted-dotted line, online blue). For $\left(\gamma_{\text {tot }}, \omega_{c}, \theta, \theta^{\prime}\right)=(0.3,10 \Delta, \pi / 4,0)$ the exponential approximation is plotted as well (black dashed line).

A careful analysis (see Appendix C) reveals that the oscillations are a consequence of the sharp cutoff function $\theta\left(\omega_{c}-\omega\right)$. To understand this on a qualitative level, we resort to a result by Viola and Lloyd, ${ }^{32}$ who investigated the decay of purity for pure dephasing of the spin with a single bath, corresponding to $\theta=0$ and $\Delta=0$. In this case, the transverse purity could be calculated exactly:

$$
\frac{\mathcal{P}_{\perp}(t)}{\mathcal{P}_{\perp}(0)}=\exp \left(-2 \int d \omega J(\omega) \frac{1-\cos (\omega t)}{\omega^{2}}\right) .
$$

This function is plotted in the right picture of Fig. 5 with $J(\omega)=2 \gamma_{\text {tot }} \omega \Theta\left(\omega_{c}-\omega\right)$ for various parameters $\left(\gamma_{\text {tot }}, \omega_{c}\right)$. Here, $\Theta(\omega)$ is Heaviside's function. The plot features oscillations with the same frequency (but with smaller amplitude) as our numerical results. The oscillations of $\mathcal{P}_{\perp}(t)$ in Eq. (36) can be smeared out by using a smooth cutoff functions such as, for instance, $\exp \left(-\omega / \omega_{c}\right)$ instead of a sharp one (see Appendix C).

Decoherence for symmetric coupling $(\theta=\pi / 4)$ and for single-bath coupling $(\theta=0)$ can not be compared unambiguously because in the latter case the decay depends strongly on the chosen initial state. The two cases $(0.3,10 \Delta, \pi / 4,0)$ (black crosses) and $(0.3,10 \Delta, 0, \pi / 4)$ (yellow triangles) are probably best suited for comparison since they have the same short-time expansion and the same decay rate in the Bloch equation. The short-time behavior is indeed similar, however, for larger times in the case of a single bath apart from $\omega_{c}^{-1}$ a second time scale shows up on which $\mathcal{P}_{\perp}$ oscillates. These oscillations occur even for the weak quantum Zeno effect. They are absent for symmetric coupling $(\theta=\pi / 4)$. Due to the oscillations with different frequencies, the transverse purity fluctuates much wilder for a coupling to a single bath than for a symmetric coupling to two baths.

The occurrence of oscillations with other frequencies than $\omega_{c}$ becomes plausible if one pushes forward the small-time expansion (Sec. IV B) to higher orders $2 \mathcal{P}_{\perp}(t)=1+p_{1} t+$ $\ldots+p_{n} t^{n}+\mathcal{O}\left(t^{n+1}\right)$. At order $t^{n}$, in principle, all combinations of the form $\Delta^{k} \omega_{c}^{n-k}$ occur in $p_{n}$ and might give rise to oscillations with frequency $\sqrt[n]{\Delta^{k} \omega_{c}^{n-k}}$. These oscillations vanish in the limit $\omega_{c} / \Delta \rightarrow \infty$.
In Fig. 6, the transverse purity is plotted for different initial states characterized by the angles $\theta^{\prime}=0, \theta^{\prime}=\pi / 4$, and $\theta^{\prime}=\pi / 2$. For a single bath, the initial decay becomes faster as the angle $\theta^{\prime}$ increases from zero (weak quantum Zeno effect) to $\pi / 2$. For symmetric coupling, $\mathcal{P}_{\perp}$ is independent of the angle $\theta^{\prime}$. This invariance is due to the $U(1)$ symmetry of the Hamiltonian for symmetric coupling, where $\lambda_{3, k}^{(1)}=\lambda_{2, k}^{(2)}$ and $\omega_{k}^{(1)}=$ $\omega_{k}^{(2)} \forall k$. The Hamiltonian (2) is invariant under the canonical transformation $S_{2} \rightarrow \cos (\phi) S_{2}-\sin (\phi) S_{3}, S_{3} \rightarrow \cos (\phi) S_{3}+$ $\sin (\phi) S_{2}$, respectively, $a_{1, k} \rightarrow \cos (\phi) a_{1, k}+i \sin (\phi) a_{2, k}$ and $a_{2, k} \rightarrow \cos (\phi) a_{2, k}+i \sin (\phi) a_{1, k}$. This transformation clearly rotates the direction of the initial spin by the angle $\phi$ in the $y-z$ plane.

The time evolution of $\left\langle S_{1}(t)\right\rangle$ is plotted in Fig. 7 for symmetric coupling $(\theta=\pi / 4)$ and for a single bath $(\theta=0)$ for the coupling strength $\gamma_{\text {tot }}=0.3$. The numerics were performed with 100 uniformly distributed bath modes. Here, $1-\left\langle S_{1}(t)\right\rangle$ decays initially faster for a single bath than

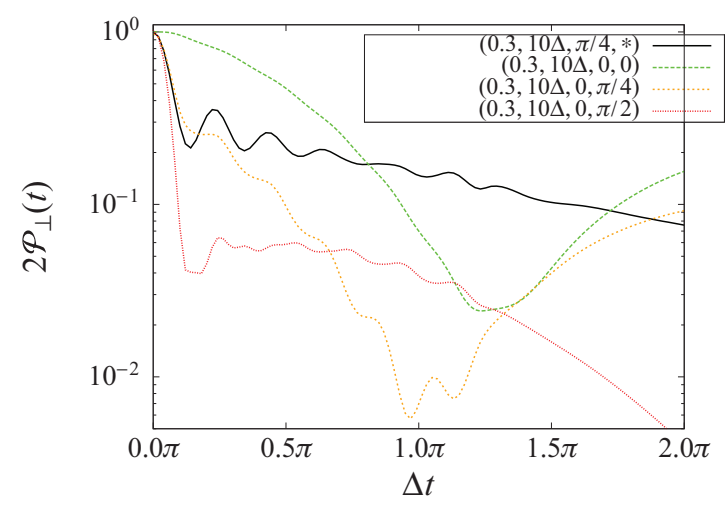

FIG. 6. (Color online) Time evolution of the transversal purity for different initial state characterized by the angle $\theta^{\prime}$ for symmetric coupling (full line, online black) and for a single bath, which couples to the spin operator in the $z$ direction $(\theta=0)$ with $\theta^{\prime}=0$ (dashed line, online green), $\theta^{\prime}=\pi / 4$ (short dashed line, online orange), and $\theta^{\prime}=\pi / 2$ (dotted line, online red). Only for a single bath there is a dependence on $\theta^{\prime}$. The other parameters are $\gamma_{\text {tot }}=0.3$ and $\omega_{c} / \Delta=10$. 


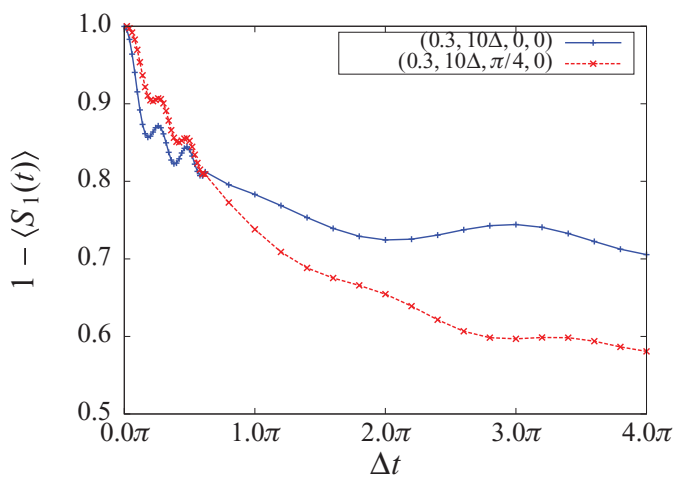

FIG. 7. (Color online) Time evolution of the expectation value $\left\langle S_{1}(t)\right\rangle$ for the initial state (27) for symmetric coupling (online red, crosses) and for a single bath (online blue, stars), $\gamma_{\text {tot }}=0.3, \omega_{c} / \Delta=$ 10.

for symmetric coupling. However, on a time scale of one Rabi oscillation, the decay grows faster for symmetric coupling to reach an equilibrium value, which is smaller than for a single bath in accordance with Fig. 1.

\section{SUMMARY AND DISCUSSION}

While the calculation of equilibrium correlation functions is somewhat cumbersome within the flow-equation approach, the method turns out to be a useful numerical tool in nonequilibrium physics. We were able to monitor purity decay on the time scale of the quantum Zeno time as well as on the time scale of the inverse Rabi frequency.

When one speaks about coherence of a two-level system, one has to distinguish carefully between the decay of the off-diagonal elements and of the diagonal elements. It is characteristic for a small-size Hilbert space that both are not independent and the distinction between decoherence and dissipation is fuzzy.

In our analysis, frustration effects of two independent oscillator baths could be identified in the renormalized energy gap $\Delta_{r}$, in the ground-state expectation value of $S_{1}$ and in the ground-state energy shift. These quantities are protected by a symmetric coupling. In particular, the protection of $\left\langle S_{1}\right\rangle_{\beta}$ can rightly be called protection of decoherence since it contributes to a high equilibrium purity of the spin.

We were able to identify a phase diagram in the plane of the two dimensionless coupling strengths $\gamma_{2}^{(2)}$ and $\gamma_{3}^{(1)}$, where a localized phase is separated from a delocalized phase by a critical line. For strong but not complete asymmetry, the delocalized phase extends deep into regions with values $\gamma_{\text {tot }}>1$. This affirms that the delocalized phase for symmetric coupling $(\theta=\pi / 4)$ and $1<\gamma_{\text {tot }}<\infty$ is stable against small asymmetries.

The flow equations (10) might be truncated by setting all second-order terms, i.e., the matrix entries of $T$ [Eq. (8)], to zero. The truncated flow equations can be analyzed analytically (see Appendix A). The outcome is $\ln \left(\Delta_{r} / \Delta\right) \propto-\gamma_{\text {tot }} /(1-$ $\left.\gamma_{\text {tot }}\right)$, similar to the old result by Silbey and Harris ${ }^{33}$ which features no dependence on the asymmetry angle. This shows that the dependence of the renormalized energy gap $\Delta_{r}$ on an asymmetry angle is a generic nonperturbative effect.
The perturbative RNG equations (18) and (19) obtained from the flow equations are completely symmetric in the four coupling constants $\gamma_{2}^{(n)}, \gamma_{3}^{(n)}, n=1,2$. Setting any two of them to zero yields the RNG equations of Ref. 1, with the implication of a delocalized phase for $\gamma_{\text {tot }} \rightarrow \infty$. Setting, for instance, $\gamma_{2}^{(2)}=\gamma_{3}^{(2)}=0$, this implies that also a symmetric coupling of the spin with its $y$ and $z$ components to a single bath can protect the delocalized phase.

Decoherence and relaxation from a nonequilibrium initial state are complicated. The decay of both transverse and parallel purity depends nonuniversally on cutoff frequency and shape of the cutoff function. In particular for a sharp cutoff, oscillations on the time scale of the cutoff frequency show up. Moreover, for an asymmetric coupling decay of decoherence depends crucially on the initial state. For a single bath, decay is fastest if the initial spin is aligned perpendicular to the spin operator, which couples to the oscillator bath and it is slowest if the spin is aligned parallel (weak quantum Zeno effect). Due to the $U(1)$ invariance of the Hamiltonian under rotations in the $y-z$ plane perpendicular to the external magnetic field, this dependence on the initial state is absent for symmetric coupling. This independence on the initial state can justifiably be interpreted as a signature of quantum frustration as defined in the Introduction. It was was already pointed out in Refs. 1 and 2 that, as an application of the Mermin-Wagner theorem, due to this $U(1)$ invariance the localization phase transition is suppressed.

On the other hand, our results clearly show that the competition of two heat baths can not prevent the fast decay of coherence of an initial product state. The purity of the reduced density matrix incurs important initial slips on the time scale of the quantum Zeno time. The quantum Zeno time itself is determined via Eq. (33) by the sum of the two integrals over the spectral functions. Thus, the addition of a second bath will reduce the quantum Zeno time and accelerate the initial decay of coherence.

In summary, the competition between two oscillator baths, which couple to noncommuting observables of the spin, leads to quite different effects in equilibrium and in nonequilibrium. In equilibrium, the noncommutativity of the spin observables induces an effective protection of equilibrium properties of the uncoupled spin, as exemplified by the ground-state energy shift or correlation functions. Here, one can rightly speak of quantum frustration. On the other hand, in nonequilibrium, in particular for the short-time dynamics, the noncommutativity of the spin observables has much lesser visible effects. The addition of a second bath will in general not lead to a better protection of the coherence of the initially prepared state.

\section{ACKNOWLEDGMENTS}

H.K. acknowledges financial support from the German Research council (DFG) with Grant No. Ko 3538/1-2 and from CSIC within the JAE-Doc program cofunded by the FSE (Fondo Social Europeo). A.H. acknowledges support by the David and Ellen Lee foundation. We acknowledge useful discussions with F. Guinea, F. Sols, and T. Stauber. The computer cluster of the University of Duisburg-Essen was used for the numerics. 


\section{APPENDIX A: LINEARIZED FLOW EQUATIONS FOR TWO BATHS}

We consider the linearized version of the flow equations. In the linearized version of the flow equations, the flow of $T$ can be neglected:

$$
\frac{d \vec{\Lambda}(B)}{d B}=-(\Delta-\Omega) \vec{\Lambda} .
$$

For Ohmic spectral functions $J_{i}^{(n)}(\omega)=2 \gamma_{i}^{(n)} \omega \theta\left(\omega_{c}-\omega\right), i=$ $2,3, n=1,2$, immediately the first-order RG equations

$$
\frac{d \gamma_{i}^{(n)}}{d B}=-\Delta^{2} \gamma_{i}^{(n)}, \quad i=2,3, \quad n=1,2
$$

are obtained. Introducing the auxiliary densities

$$
G_{ \pm}^{(n)}(\omega)=\sum_{k}\left(\lambda_{ \pm, k}^{(n)}\right)^{2} \delta\left(\omega-\omega_{k}^{(n)}\right), \quad n=1,2
$$

the renormalization group equation for the tunneling matrix element (10) can be written as

$$
\frac{d \Delta(B)}{d B}=-\frac{1}{4} \sum_{n=1}^{2} \sum_{\sigma= \pm} \int d \omega \frac{\operatorname{coth}(\beta \omega / 2)}{\Delta(B)-\sigma \omega} \frac{d}{d B} G_{\sigma}^{(n)}(\omega, B) .
$$

Following the outlines of Ref. 20, a self-consistency equation for $\Delta_{r}$ can be obtained. For zero temperature it reads as

$$
\ln \frac{\Delta_{r}}{\Delta}=\sum_{n=1}^{2} \sum_{\sigma= \pm} \int_{0}^{\infty} \frac{d \omega}{4 \Delta_{r}} \frac{G_{\sigma}^{(n)}(\omega, 0)}{\Delta_{r}-\sigma \omega} .
$$

For $\lambda_{2, k}^{(1)}=\lambda_{3, k}^{(2)}=0, G_{+}^{(1)}=G_{-}^{(1)}=J_{3}^{(1)}$, and $G_{+}^{(2)}=G_{-}^{(2)}=$ $J_{2}^{(2)}$ and for an Ohmic bath the renormalized matrix element becomes

$$
\Delta_{r}=\Delta\left(\frac{\Delta}{\omega_{c}}\right)^{\frac{\gamma_{3}^{(1)}+\gamma_{2}^{(2)}}{1-\gamma_{2}^{(1)}-\gamma_{3}^{(2)}}} .
$$

This is a straightforward extension of the old result by Silbey and Harris. ${ }^{33}$ In the linear approximation of the flow equations there is no angle dependence of $\Delta_{r}$. The full flow equations must be employed.

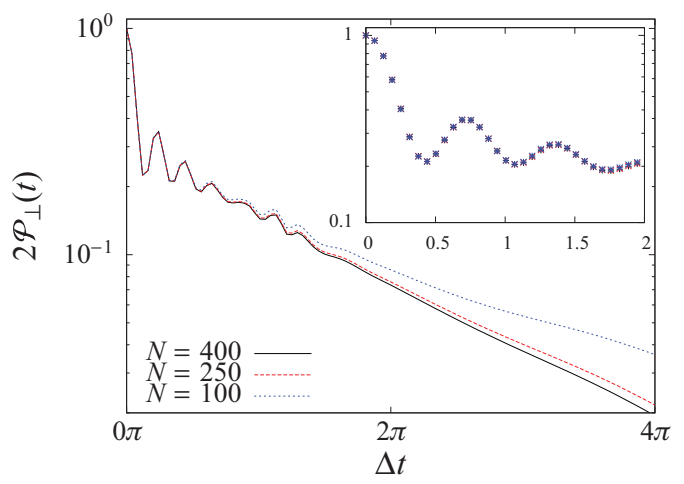

\section{APPENDIX B: FLOW EQUATIONS FOR THE SPIN OPERATORS}

The flow equations for the expansion coefficients of the spin operators are obtained from the commutators $\left[\eta, S_{1}\right]$ and $\left[\eta, S_{ \pm}\right]$. They read as

$$
\begin{aligned}
\frac{d h_{0}(B)}{d B} & =\frac{1}{2} \vec{\Lambda}^{T}(\Delta-\Omega) \Sigma_{z} \vec{\chi}, \\
\frac{d h_{1}(B)}{d B} & =-\frac{1}{2} \vec{\Lambda}^{T}(\Delta-\Omega) \operatorname{coth}\left(\frac{\beta|\Omega|}{2}\right) \vec{\chi}, \\
\frac{d h_{+}(B)}{d B} & =\frac{1}{2}\left[\vec{\zeta}_{1}^{\dagger} \operatorname{coth}\left(\frac{\beta|\Omega|}{2}\right)-\vec{\zeta}_{0}^{\dagger} \Sigma_{z}\right] \Sigma_{x}(\Delta-\Omega) \vec{\Lambda}, \\
\frac{d h_{-}(B)}{d B} & =\frac{1}{2}\left[\vec{\zeta}_{1}^{\dagger} \operatorname{coth}\left(\frac{\beta|\Omega|}{2}\right)+\vec{\zeta}_{0}^{\dagger} \Sigma_{z}\right](\Delta-\Omega) \vec{\Lambda}, \\
\frac{d \vec{\chi}(B)}{d B} & =h_{1}(\Delta-\Omega) \vec{\Lambda}+[\Omega, T] \operatorname{coth}\left(\frac{\beta|\Omega|}{2}\right) \vec{\chi}, \\
\frac{d \vec{\zeta}_{0}(B)}{d B} & =+[\Omega, T] \Sigma_{z} \vec{\zeta}_{1}, \\
\frac{d \vec{\zeta}_{1}(B)}{d B} & =-\frac{1}{2}\left(h_{-}^{*}+h_{+}^{*} \Sigma_{x}\right)(\Delta-\Omega) \vec{\Lambda}+[\Omega, T] \Sigma_{z} \vec{\zeta}_{0} .
\end{aligned}
$$

These differential equations are the same for the forward flow and for the backward flow. However, the initial conditions are different. For the forward flow, the initial conditions are $h_{1}=h_{+}=1$ and all other components are zero. Since the differential equations are linear in the expansion coefficients the imaginary parts of $h_{ \pm}, \vec{\chi}$, and $\vec{\zeta}_{0,1}$ remain zero throughout the flow.

Due to the time evolution, the imaginary parts acquire a nontrivial backward flow. The initial conditions are now $\operatorname{Re} \overrightarrow{\tilde{\chi}}(t, 0)=\cos \left[\left(\Omega-\Delta_{r}\right) t\right] \operatorname{Re} \vec{\chi}(\infty), \operatorname{Im} \overrightarrow{\tilde{\chi}}(t, 0)=$ $\sin \left[\left(\Omega-\Delta_{r}\right) t\right] \operatorname{Re} \vec{\chi}(\infty), \operatorname{Re} \overrightarrow{\tilde{\zeta}}_{0,1}(t, 0)=\cos (\Omega t) \operatorname{Re} \vec{\zeta}_{0,1}(\infty)$, $\operatorname{Im} \overrightarrow{\tilde{\zeta}}_{0,1}(t, 0)=\operatorname{Re} \vec{\zeta}_{0,1}(\infty) \sin (\Omega t), \operatorname{Re} \tilde{h}_{ \pm}(t, 0)=\cos \left(\Delta_{r} t\right)$ $h_{ \pm}(\infty)$, and $\operatorname{Im} \tilde{h}_{ \pm}(t, 0)= \pm \sin \left(\Delta_{r} t\right) \operatorname{Re} h_{ \pm}(\infty)$. The flow of the imaginary parts $\operatorname{Im} \overrightarrow{\tilde{\chi}}$ decouples from that of the real parts and of $h_{0}$ and of $H_{1}$. Thus, it needs not to be considered.

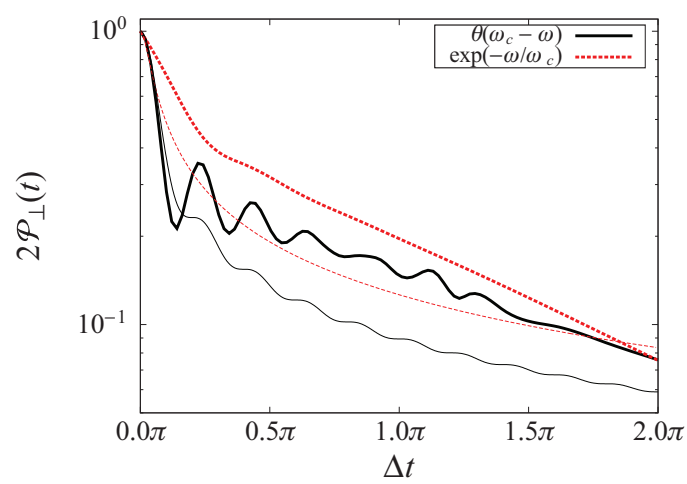

FIG. 8. (Color online) Left: Transverse purity $\mathcal{P}_{\perp}$ for different numbers of bath modes $N=400,250$, and 100 for symmetric coupling and $\gamma_{\text {tot }}=0.3, \omega_{c} / \Delta=10$. In the inset, the raw data points are plotted for short times. There is no visible difference in all three cases. Right: $\mathcal{P}_{\perp}$ for a sharp cutoff (full line, online black) and for an exponential cutoff (dashed line, online red). For comparison, in thinner lines the function $\mathcal{P}_{\perp}$ of Eq. (36) is plotted, showing the same behavior. 


\section{APPENDIX C: NUMERICAL DETAILS OF THE NONEQUILIBRIUM CALCULATIONS}

In order to get an estimate of the numerical error, we calculated $\mathcal{P}_{\perp}$ for different numbers $N(N=100,250$, and 400) of bath modes, which were uniformly distributed between zero and $\omega_{c}=10 \Delta$. Therefore, $\omega_{k}^{(1)}=k \omega_{c} / N, 1 \leqslant k \leqslant N$, $k \in \mathbb{N}$ (for the relation between $\omega_{k}^{(1)}$ and $\omega_{k}^{(2)}$, see below). The other parameters were $\gamma_{\text {tot }}=0.3, \theta=\pi / 4$ (symmetric coupling), and $\theta^{\prime}=0$. Thus, the coupling coefficients were given by $\lambda_{3, k}^{(1)}=\sqrt{\omega_{k}^{(1)} \gamma_{\text {tot }} \omega_{c} /(N+1)}$ and by $\lambda_{2, k}^{(2)}=$ $\sqrt{\omega_{k}^{(2)} \gamma_{\text {tot }} \omega_{c} /(N+1)}$

On the left-hand side of Fig. 8, the transverse purity $\mathcal{P}_{\perp}$ is plotted. Whereas for small times there is no visible difference for the different numbers of bath modes, for times of order of one Rabi oscillation effects of the bath discretization become visible. This is much sooner than Heisenberg time $2 \pi N / \omega_{c}$.
A calculation with linearly decreasing density of bath modes $\omega_{k}^{(n)}=k^{2} \omega_{c} / N^{2}, 1 \leqslant k \leqslant N, k \in \mathbb{N}$, was also performed for $N=250$. In the plots (not shown here) no visible difference existed to the case of uniformly distributed bath modes for times up to two Rabi oscillations. On the right, $\mathcal{P}_{\perp}$ is compared for a sharp cutoff function $J_{2}^{(2)}(\omega)=$ $J_{3}^{(1)}(\omega)=\gamma_{\text {tot }} \omega \theta\left(\omega_{c}-\omega\right)$ for $N=250$ modes, which are uniformly distributed between zero and $\omega_{c}=10 \Delta$ and for an exponential cutoff $J(\omega)=\gamma_{\text {tot }} \omega \theta\left(\omega_{c}-\omega\right)$. For the latter, 400 modes were uniformly distributed between zero and $4 \omega_{c}$. It is seen that the oscillations are smeared out.

In our numerics, we used frequencies $\omega_{k}^{(2)}=\omega_{k}^{(1)} \pm \epsilon$ with a fixed offset $\epsilon$ of order of the mean level spacing. Thus, the frequencies of the two oscillator baths were correlated. Strictly speaking, in the model defined by the Hamiltonian (2) the bath modes are uncorrelated. This can be achieved by replacing $\epsilon$ by a uniformly distributed random number in an interval of the order of the mean level spacing. We checked numerically that the effects of this replacement are negligible. *hkohler@icmm.csic.es

${ }^{1}$ A. H. Castro Neto, E. Novais, L. Borda, G. Zarand, and I. Affleck, Phys. Rev. Lett. 91, 096401 (2003).

${ }^{2}$ E. Novais, A. H. Castro Neto, L. Borda, I. Affleck, and G. Zarand, Phys. Rev. B 72, 014417 (2005).

${ }^{3}$ C. Guo, A. Weichselbaum, J. von Delft, and M. Vojta, Phys. Rev. Lett. 108, 160401 (2012).

${ }^{4}$ D. D. Bhaktavatsala Rao, H. Kohler, and F. Sols, New J. Phys. 10, 115017 (2008).

${ }^{5}$ H. Kohler and F. Sols, Phys. Rev. B 72, 180404 (2005).

${ }^{6}$ H. Kohler and F. Sols, New J. Phys. 8, 149 (2006).

${ }^{7}$ A. Cuccoli, N. DelSette, and R. Vaia, Phys. Rev. E 81, 041110 (2010).

${ }^{8}$ H. Kohler and F. Sols, Phys. A (Amsterdam) 392, 1989 (2013).

${ }^{9}$ A. Cuccoli, A. Fubini, V. Tognetti, and R. Vaia, Path Integrals: New Trends and Perspectives (World Scientific, Singapore, 2008), p. 500.

${ }^{10}$ D. Giuliano and P. Sodano, New J. Phys. 10, 093023 (2008).

${ }^{11}$ N. Erez, G. Gordon, M. Nest, and G. Kuritzki, Nature (London) 452, 724 (2008).

${ }^{12}$ L. Zhu and Q. Si, Phys. Rev. B 66, 024426 (2002).

${ }^{13}$ G. Zárand and E. Demler, Phys. Rev. B 66, 024427 (2002).

${ }^{14}$ P. W. Anderson and G. Yuval, J. Phys. C: Solid State Phys. 4, 607 (1971).
${ }^{15}$ A. J. Bray and M. A. Moore, Phys. Rev. Lett. 49, 1545 (1982).

${ }^{16}$ C. P. Slichter, Principles of Magnetic Resonance, 3rd ed., Springer Series in Solid State Sciences 1 (Springer, Heidelberg, 1996).

${ }^{17}$ F. Haake and R. Reibold, Phys. Rev. A 32, 2462 (1985).

${ }^{18}$ S. D. Głazek and K. G. Wilson, Phys. Rev. D 48, 5863 (1993).

${ }^{19}$ F. Wegner, Ann. Phys. (Leipzig) 3, 77 (1994).

${ }^{20}$ S. Kehrein, The Flow Equation Approach to Many Particle Systems, 1st ed. (Springer, Heidelberg, 2006).

${ }^{21}$ S. Kehrein, A. Mielke, and P. Neu, Z. Phys. B 99, 269 (1996).

${ }^{22}$ S. Kehrein and A. Mielke, Phys. Lett. A 219, 313 (1996).

${ }^{23}$ S. Kehrein and A. Mielke, Ann. Phys. (Leipzig) 6, 90 (1997).

${ }^{24}$ T. Stauber and A. Mielke, Phys. Lett. A 305, 275 (2002).

${ }^{25}$ A. Hackl and S. Kehrein, Phys. Rev. B 78, 092303 (2008).

${ }^{26}$ A. Hackl and S. Kehrein, J. Phys.: Condens. Matter 21, 015601 (2009).

${ }^{27}$ F. Wegner, J. Phys. A: Math. Gen. 39, 8221 (2006).

${ }^{28}$ T. Stauber and A. Mielke, J. Phys. A: Math. Gen. 36, 2707 (2003).

${ }^{29}$ P. Facchi and S. Pascazio, Phys. Lett. A 241, 139 (1998).

${ }^{30}$ W. M. Itano, D. J. Heinzen, J. J. Bollinger, and D. J. Wineland, Phys. Rev. A 41, 2295 (1990).

${ }^{31}$ B. Misra and E. Sudarshan, J. Math. Phys. 18, 756 (1977).

${ }^{32}$ L. Viola and S. Lloyd, Phys. Rev. A 58, 2733 (1998).

${ }^{33}$ R. Silbey and R. A. Harris, J. Chem. Phys. 80, 2615 (1983). 